PHYSICAL REVIEW D 93, 029901(E) (2016)

\title{
Erratum: Post-Newtonian constraints on Lorentz-violating gravity theories with a MOND phenomenology [Phys. Rev. D 91, 084053 (2015)]
}

\author{
Matteo Bonetti and Enrico Barausse \\ (Received 31 December 2015; published 13 January 2016)
}

DOI: 10.1103/PhysRevD.93.029901

We have found a few typos in our article, which we correct below. Since these typos are not present in our actual calculations, they do not propagate anywhere, and the results of the paper are unaffected.

In more detail, the right-hand side of Eq. (18) has a wrong sign; i.e. the correct equation reads

$$
D_{j}\left(K^{i j}-\frac{1+\lambda}{1-\beta} \gamma^{i j} K\right)=-\frac{8 \pi G \mathcal{J}^{i}}{(1-\beta) c^{4}} .
$$

Also, in the second line of Eq. (19), a $K$ is missing; i.e. the correct equation is

$$
\begin{gathered}
\frac{1}{1-\beta}\left({ }^{(3)} R^{i j}-\frac{1}{2}{ }^{(3)} R \gamma^{i j}\right)+\frac{1}{N} D_{t}\left(K^{i j}-\frac{1+\lambda}{1-\beta} \gamma^{i j} K\right) \\
+\frac{2}{N} D_{k}\left(N^{(i}\left(K^{j) k}-K \frac{1+\lambda}{1-\beta} \gamma^{j) k}\right)\right)+2 K^{i k} K_{k}^{j} \\
-\frac{1+\beta+2 \lambda}{1-\beta} K^{i j} K-\frac{1}{2} \gamma^{i j}\left(K^{k l} K_{k l}+\frac{1+\lambda}{1-\beta} K^{2}\right) \\
-\frac{1}{(1-\beta) N}\left(D^{i} D^{j} N-\gamma^{i j} D_{k} D^{k} N\right) \\
+\frac{1}{1-\beta} \chi a^{i} a^{j}-\frac{f(a)}{2(1-\beta)} \gamma^{i j}=\frac{8 \pi G}{(1-\beta) c^{4}} \mathcal{T}^{i j} .
\end{gathered}
$$

Moreover, right before Eq. (26), in the sentence "obtained by contracting that equation with $\gamma^{i j}$," the 3-metric should have covariant indices; i.e. the sentence should read "obtained by contracting that equation with $\gamma_{i j}$."

Finally, in footnote 4, the relation between $\Lambda$ and $\Lambda_{\mathrm{obs}}$ is actually $\Lambda=\Lambda_{\mathrm{obs}} G / G_{c}$. However, the meaning of the footnote, which was supposed to show that $\Lambda \sim \Lambda_{\text {obs }}$, is unaffected. In more detail, the corrected footnote should read " $\Lambda$ is related to the measured cosmological constant $\Lambda_{\mathrm{obs}}$ by $\Lambda=\Lambda_{\mathrm{obs}} G / G_{c}$, where $G_{c}=2 G /(2+\beta+3 \lambda)$ is the gravitational constant appearing in the Friedmann equations [1]. In practice, since BBN metal abundances and binary-pulsar observations constrain $|\beta, \lambda| \lesssim$ a few $\times 0.01[2,3]$ (cf. also Sec. III), it must be $\Lambda \sim \Lambda_{\text {obs." }}$

We thank Daniele Vernieri and Noemi Frusciante for bringing this last typo to our attention.

[1] D. Blas, M. M. Ivanov, and S. Sibiryakov, J. Cosmol. Astropart. Phys. 10 (2012) 057.

[2] K. Yagi, D. Blas, E. Barausse, and N. Yunes, Phys. Rev. D 89, 084067 (2014).

[3] K. Yagi, D. Blas, N. Yunes, and E. Barausse, Phys. Rev. Lett. 112, 161101 (2014). 